$R M x A C, \mathbf{5 3}, 42-43(2021)$

(c) 2021: Instituto de Astronomía, Universidad Nacional Autónoma de México

https://doi.org/10.22201/ia.14052059p.2021.53.10

\title{
INTRODUCING OBSERVATORY OF IRANIAN SPACE AGENCY MAHDASHT SPACE CENTER
}

\author{
S. H. Hendi ${ }^{1,2}$ and F. Bahrani ${ }^{1,3}$
}

\section{RESUMEN}

Como los observatorios robóticos son cada vez más y más populares, en algunos emplazamientos llegan a ser casi obligatorios. En este sentido, la agencia espacial iraní está desarrollando el primer observatorio robótico en Irán. Aunque el telescopio del centro espacial de Mahdasht es robótico, todavía se necesita un desarrollo adicional para lograr hacer su cúpula robótica también. Se presentan los trabajos encaminados para su consecución.

\section{ABSTRACT}

As robotic observatories getting more and more popular, it becomes mandatory for some places. In this regard, Iranian space agency trying to build the first robotic observatory in Iran. Although the telescope of Mahdasht space center is robotic at this time, it still needs more attention to make its dome robotic too. In this article, we introduce this space center and its development plan.

Key Words: telescopes

\section{INTRODUCTION}

Robotic telescopes help astronomers to obtain their data in a simple manner with more quality in less time. The observatory of Mahdasht space center, a robotic telescope belongs to Iranian Space Agency, was built in 2011. It is located in the longitude of eastern $50^{\circ} 47^{\prime} 8.58^{\prime \prime}$ and latitude of northern $35^{\circ}$ $45^{\prime} 47.34^{\prime \prime}$ and altitude of 1198 meter, and has a dome with 4 meter diameter.

\section{EQUIPMENT}

The main telescope of Mahdasht space center observatory is a 16 inches Schmidt - Cassegrain model of Mead LX 200 which is on Paramount Me SynScan mount that located under a dome with a diameter of 4 meter. The main telescope has a system of automatic search and can connect to computer and CCD to take professional images. Here, we mention other equipment of Mahdasht space center observatory:

- A 16 inches Dobsonian SkyWatcher telescope.

- A 6 inches Newtonian SkyWatcher telescope.

- A 90 millimeter solar telescope, model of Coronado Solarmax II.

- A 120 millimeter apochromatic reflecting SkyWatcher telescope.

\footnotetext{
${ }^{1}$ Physics Department and Biruni Observatory, College of Sciences, Shiraz University, Shiraz 71454, Iran

${ }^{2}$ Hendi@shirazu.ac.ir

${ }^{3}$ Bahrani-f@yahoo.com
}

- A 80 millimeter achromatic reflecting SkyWatcher telescope.

- An $20 * 80$ and a $25 * 100$ binocular, model of NightSky.

- A CCD imaging camera model of SBIG STL$11000 \mathrm{CM}$.

- A CCD video camera model of Astrovid Stellacam 3.

- A Canon imaging camera model of 5D Mark II.

- Filters for observation of the moon, sun and nebulae.

- 50 millimeter filter set.

- Camera charger.

- Leaser aligning device for Cassegrain telescopes.

- Books, magazines and atlas related to astronomy.

\section{FACILITIES AND ACTIVITIES}

Except for the internet, library and suits for staying astronomers during observation nights, the main facility of this observatory is its warm room which allows astronomers to control observatory robotic telescope by using of internet. The Mahdasht space center has a special training program for the public once a week. In this program, during two hours, one 


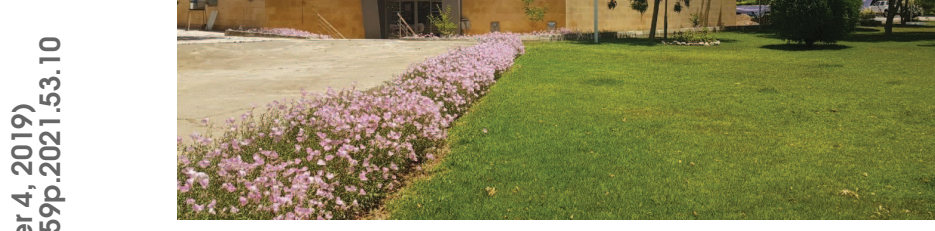

Fig. 1. A view of Mahdasht space center observatory.

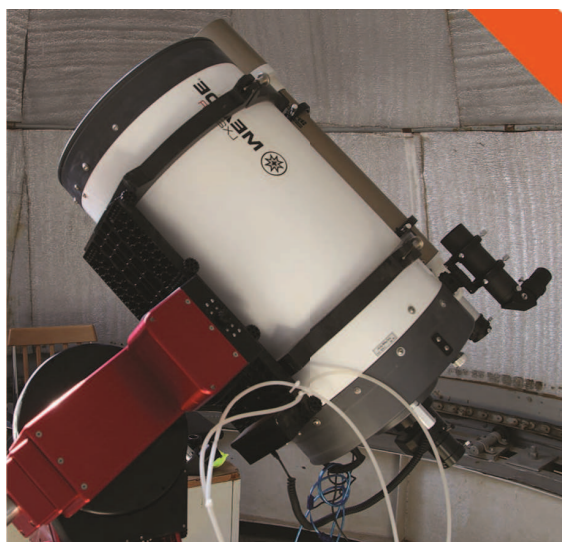

Fig. 2. 16 inches Schmidt - Cassegrain telescope.

introduces astronomical software, teaches basic concepts of astronomy, shows films and pictures, demonstrates the main telescope with a short time observation. Besides, for researchers, the observatory provides wider range of national and international support. Astronomers can ask data from observatory and monitor collection of their needed data by internet or do observation by themselves with the tele-

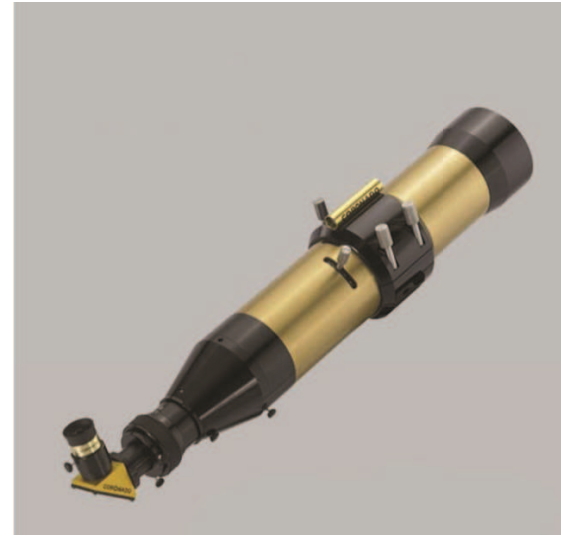

Fig. 3. 90 millimeter solar telescope.

scope of observatory. Also, Mahdasht space center observatory supports workshops, training schools and etc by offering the facilities of observatory.

\section{FUTURE PLAN}

As we mentioned before, the main telescope and its equipment are robotic but automatization of the dome still need to make robotic which is under progress plan. In addition, one can propose to change its location to a more suitable place. Furthermore, it is suggested to provide another high resolution telescope.

Acknowledgements: We thank Ramin Poureh from Iranian Space Agency for helping us with his useful information.

\section{REFERENCES}

Pepper, J., Pogge, R. W., DePoy, D. L., et al. 2007, PASP, 119, 923

Gomez, E. L. \& Fitzgerald, M. T. 2017, AstRv, 13, 28

Tarikhi, P. 2014, The Iranian Space Endeavor: Ambitions and Reality, Springer

Harvey, B., Smid, H. F., Pirard, T. 2010, Emerging Space

Powers, Praxis, pp 255-284 